\section{Delirum: Antipsychotika verschlechtern die Stress-Symptome bei Palliativpatienten}

\author{
Hydratation, Reorientierung und soziale Unterstützung durch Familien- \\ angehörige sind die wichtigsten Supportivmaßnahmen bei Delirium. \\ Können Antipsychotika Disstress-Symptome zusätzlich abschwächen?
}

\begin{abstract}
D isstress und Verhaltensauffälligkeiten im Rahmen eines Deliriums werden häufig mit Antipsychotika behandelt. Kontrollierte Studien zeigten eine Verbesserung der deliranten Symptomatik, allerdings auf methodisch nicht sehr hohem Niveau. Leitlinien empfehlen Antipsychotika lediglich für die Behandlung von starkem Disstress und Verhaltensstörungen, die auf andere Maßnahmen nicht ansprechen. Eine große placebokontrollierte Studie sollte nun die stressreduzierenden Effekte von Risperidon und Haloperidol bei Delirpatienten in der Palliativsituation untersuchen.

In der prospektiven randomisierten Studie wurden 247 Palliativpatienten
\end{abstract}

untersucht, die doppelblind, altersadjustiert und dosistitriert entweder orales Risperidon, orales Haloperidol oder Placebo erhielten. Die Medikation wurde über 72 Stunden gegeben, bei Bedarf war Midazolam erlaubt. Zusätzlich wurden die Patienten mit Allgemeinmaßnahmen wie Hydratation sowie Seh-, Hörund Reorientierungshilfen unterstützt. Primäres Studienziel war die durchschnittliche Gruppendifferenz des Delirium-Symptomscores, berechnet als Summe der Verhaltens-, Kommunikations- und Wahrnehmungsitems aus der „Nursing Delirium Screening Scale" zu Behandlungsbeginn und -ende.

Das Ergebnis ist überraschend: Statt die Symptome zu bessern, verschlechter- ten beide Neuroleptika den Disstress im Vergleich zu Placebo: Im RisperidonArm stieg der Delirium-Score um durchschnittlich 0,48 Einheiten (95\%-Konfidenzintervall [KI]: 0,09-0,86; $\mathrm{p}=0,02$ ), im Haloperidol-Arm um durchschnittlich 0,24 Einheiten (95\%-KI: 0,06-0,42; $\mathrm{p}=0,009$ ). Der Midazolam-Verbrauch war in allen drei Gruppen vergleichbar. Extrapyramidale Nebenwirkungen traten bei den Neuroleptika-behandelten Patienten erwartungsgemäß häufiger auf als unter Placebo.

Fazit: Bei Palliativpatienten mit deliriumbedingtem psychischen Distress verschlechtern Risperidon und Haloperidol im Vergleich zu Placebo die Symptome und erhöhen die Rate extrapyramidaler Störungen. Die Patienten sollten daher primär eine Supportivtherapie erhalten.

Dr. Barbara Kreutzkamp

Agar MR. Efficacy of oral risperidone, haloperidol, or placebo for symptoms of delirium among patients in palliative care. A randomized clinical trial. JAMA Intern Med 2017;177:34-42

\title{
Für akutes Kreuzweh kann das Wetter nichts
}

\begin{abstract}
In einer vor zwei Jahren publizierten Studie hatten australische Forscher keinen klinisch bedeutsamen Zusammenhang zwischen Rückenschmerzen und dem Wetter finden können. Der Aufschrei Betroffener war groß. Nun ist die Untersuchung wiederholt worden. Das Ergebnis ist das gleiche.
\end{abstract}

\footnotetext{
A n der Studie, geleitet von Daniel Steffens von der Universität Sydney, waren 981 Patienten mit einer frisch aufgetretenen Episode von lumbalen Rückenschmerzen beteiligt. Analysiert wurden die offiziellen Daten des australischen Amtes für Meteorologie, und zwar zum einen die durchschnittlichen Wetterwerte am Tag vor Beginn der Beschwerden, zum anderen die Veränderungen der Wetterlage zwei Tage bis einen Tag vor dem Schmerzbeginn. Zur Kontrolle dienten die Zeiträume eine Woche und einen Monat vor dem kritischen Zeitfenster.

Weder für die Niederschlagssituation noch für die Luftfeuchtigkeit und auch nicht für die Windverhältnisse oder den Luftdruck war ein Zusammenhang mit
}

dem Einsetzen von Kreuzweh festzustellen. Allenfalls gingen wärmere Temperaturen mit einer marginal höheren Schmerzhäufigkeit einher, die sich in einer Steigerung der Odds Ratio um $20 \%$

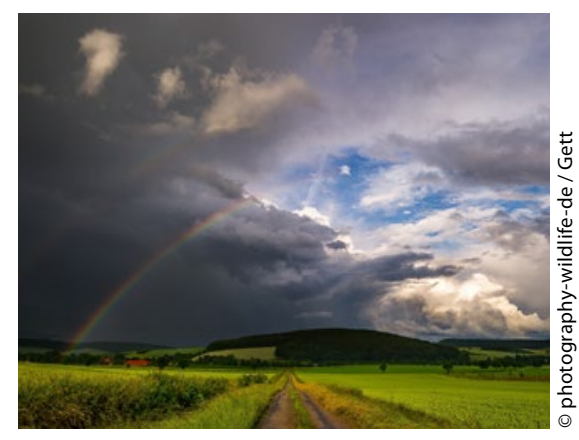

Wetterkapriolen verursachen keine akuten Rückenschmerzen. je Zunahme um $5^{\circ} \mathrm{C}$ ausdrückte und knapp die Signifikanzschwelle unterschritt $(\mathrm{p}=0,03)$. Die Forscher halten den Befund aber für klinisch irrelevant.

Die Autoren um Steffens räumen ein, es habe sich bei den Probanden um Patienten mit akuten Rückenschmerzen gehandelt. Deshalb sei das Resultat nicht ohne Weiteres auf Menschen mit chronischen Schmerzen oder Arthritis übertragbar. Deren Wetterfühligkeit zu erforschen, bleibe künftigen Studien vorbehalten.

Den Zusammenhang zwischen der Wetterlage und Rückenschmerzen hatte Steffens bereits vor zwei Jahren untersucht (Arthritis Care Res 2014;66:186772). Auch damals war das Ergebnis groBenteils negativ ausgefallen, auch wenn sich für Böigkeit und Geschwindigkeit des Windes signifikante Assoziationen ergaben. Klinisch relevant waren die Effekte aber nicht. Dr. Robert Bublak

Beilken K et al. Acute Low Back Pain? Do Not Blame the Weather-A Case-Crossover Study. Pain Med 2016 Dec 15 